\title{
Primera convulsión en niños
}

\author{
First seizure in children \\ Sergio Daniel Quintanilla,* Mario Velásquez.**
}

\section{RESUMEN}

Introducción: Las convulsiones son la urgencia neurológica más frecuente en pediatría. 10\% de la población tendrá un episodio convulsivo en algún momento de su vida. Objetivo: Determinar mediante la relación clínica, laboratorial, electroencefalográfica e imagenológica las causas de la primera convulsión en niños del Instituto Hondureño de Seguridad Social (I.H.S.S.) de San Pedro Sula, Honduras, durante el período junio 2013 a septiembre 2014. Pacientes y Métodos: Se realizó un estudio descriptivo longitudinal en el I.H.S.S., con un universo de 16930 niños atendidos en área de emergencia en el período descrito, la muestra fueron 40 niños que asistieron por primera convulsión, con edades comprendidas entre un mes y 12 años. Los pacientes fueron valorados por neurólogo pediatra o pediatra, en emergencia y/o sala de Hospitalización. Resultados: La primera convulsión se presentó en menores de dos años en el $72.5 \%$ de los casos $(n=29)$. Los principales diagnósticos fueron el síndrome convulsivo febril en $45 \% \quad(n=18)$ y epilepsia en $35 \%(n=14)$ de los casos. Se realizaron 19 electroencefalogramas obteniendo 4 con hallazgos anormales y 20 tomografías cerebrales, 5 con hallazgos anormales. Se hospitalizaron $85 \%$ de los niños con primera convulsión y en $65 \%$ (26) de los niños se inició terapia anticomicial oral de mantenimiento. Conclusiones: La población con riesgo de presentar primera convulsión son los menores de 2 años. Las causas principales de primera convulsión son las convulsiones febriles y la epilepsia.

\section{PALABRAS CLAVE}

Convulsiones, convulsiones febriles, estado epiléptico.

*Residente de Tercer año, Posgrado de Pediatría, Universidad Nacional Autónoma de Honduras en el Valle de Sula.

**Neurólogo Pediatra. Hospital Regional del Norte, Instituto Hondureño de Seguridad Social.

Dirigir correspondencia:dr.sergioquintanilla@hotmail.com

Recibido: 14 de diciembre 2015, Aprobado: 25 de mayo 2015

\section{ABSTRACT}

Introduction: Seizures are the most common pediatric neurological emergency. $10 \%$ of the population will have a seizure episode at some point in their lives. Objective: To determine by the causes of the first seizure in children at the Honduran Social Security Institute (I.H.S.S.) in San Pedro Sula during the period between June 2013 and September 2014. Patients and Methods: A longitudinal descriptive study was conducted. 16930 children attended the emergency area in the described period. The sample were 40 children between 1 month to 12 years who attended with their first seizure. Patients were evaluated by a pediatric neurologist or pediatrician. Results: The age onset of the first seizure was under two years $72.5 \%(n=29)$. The main diagnoses were febrile seizure syndrome in $45 \%(n=18)$ and epilepsy in 35\% $(n=14)$ cases. 19 EEG were obtained, 4 EEG were abnormal. Of the 20 brain scans obteined, five were abnormal. $85 \%$ of children were hospitalized due to their first seizure and $65 \%(n=26)$ of children recieved oral anticonvulsant therapy. Conclusions: The population at risk of first seizure are children under two years. The main causes of first seizure are febrile seizures and epilepsy.

\section{KEYWORDS}

Seizures, Seizures Febrile, status epilepticus.

\section{INTRODUCCIÓN}

Las convulsiones son la urgencia neurológica más frecuente en pediatría. Aproximadamente 120.000 niños tienen su primera crisis convulsiva en los Estados Unidos cada año; además, cerca del $10 \%$ de la población tendrá un episodio convulsivo en algún momento de su vida y del 2 al $4 \%$ tendrá recurrencia o presentará epilepsia. ${ }^{(1-3)}$ 
Las crisis convulsivas son descargas eléctricas anormales que tienen manifestaciones clínicas variadas, de origen multifactorial, y que se asocian a trastornos clínicos. Anteriormente, se consideraba como estatus convulsivo aquella convulsión que duraba más de 30 minutos; en algunos estudios posteriores se consideraba estatus a la duración mayor de 15 minutos. Recientemente se ha propuesto que se considere status tras 5 minutos de crisis continua o si se producen dos crisis seguidas sin recuperación entre ellas. ${ }^{(4)}$

Las primeras convulsiones se pueden clasificar como provocada y no provocada. La Primo convulsión no provocada es aquella que se presenta en un niño por primera vez y que no está relacionada a fiebre, desórdenes hidroelectrolíticos, traumatismo de cráneo reciente, $u$ otra causa reconocida. Puede ser de causa genética, estructural/metabólica, o desconocida. ${ }^{(5)}$ En la Primo convulsión provocada la crisis puede ocurrir en sujetos no epilépticos bajo la influencia de factores provocadores extra cerebrales, como hipertermia, hipoglicemia, u otras alteraciones esporádicas, transitorias y en que no está indicado dejar tratamiento crónico. ${ }^{(6)}$ El abordaje del niño que convulsiona por primera vez es muy variable, a menudo se somete al niño a una serie de exámenes que resultan incomodos, molestos, pueden acarrear altos costos, o conllevar algún riesgo (como la punción lumbar); siendo en la mayoría de los casos innecesarios y no proporcionan ninguna utilidad para todos los casos. No existen criterios universales en cuanto a la decisión de cuando iniciar medicamentos anticomiciales de mantenimiento para el niño que convulsiona por primera vez.

El presente estudio, se realizó con el objetivo de determinar mediante la relación clínica, laboratorial, electroencefalográfica e imagenológica las causas de la primera convulsión en niños del Instituto Hondureño de Seguridad Social (I.H.S.S.) de San Pedro Sula, Honduras, durante el período junio 2013 a septiem- bre 2014. Durante el proceso, se observó la necesidad de realizar un algoritmo para el abordaje estandarizado del niño con primera convulsión.

\section{PACIENTES Y MÉTODOS}

Se realizó un estudio de tipo descriptivo longitudinal, con un universo de 16,930 niños atendidos en área de emergencia del I.H.S.S. en el período de junio 2013 a septiembre del 2014; la muestra fueron 40 niños que asistieron por primera convulsión, con edades comprendidas entre un mes y 12 años. Se tomaron niños de estas edades debido a que 12 años es la edad límite de afiliación de niños; no se incluyeron menores de un mes pues la patología en este grupo de edad es muy variada y las convulsiones neonatales son tema de estudio aparte. Todos los pacientes fueron valorados por Neurólogo Pediatra y/o Pediatra, en emergencia o sala de hospitalización. Los criterios de inclusión en el estudio fueron tener edad entre 1 mes y 12 años, asistir a consulta por presentar primer episodio de convulsión, paciente captado en emergencia u hospitalizado por primera convulsión y que los padres/tutor brindaran consentimiento informado. Los criterios de exclusión fueron niños menores de 1 mes de edad o mayor de 12 años; diagnóstico previo de epilepsia, parálisis cerebral infantil, tumor cerebral, hidrocefalia o microcefalia; tampoco se incluyeron pacientes que utilizaban previamente anticonvulsivantes por cualquier causa. (Ver Diagrama No. 1.)

\section{Diagrama No. 1: Diagrama de flujo de pacientes enrolados en el estudio.}

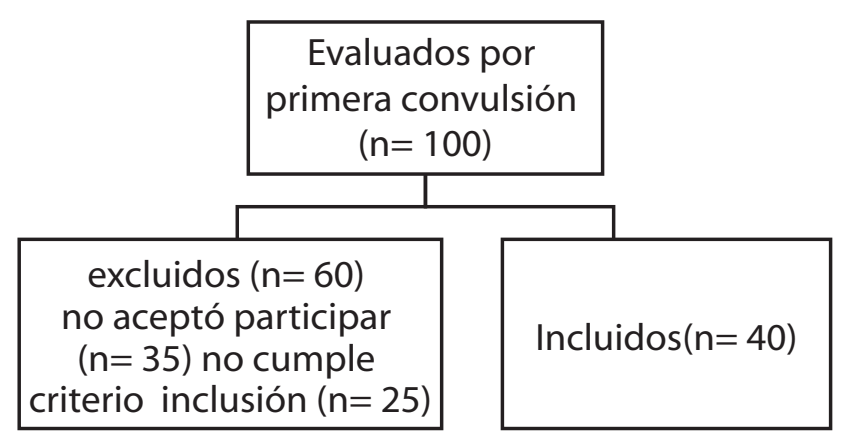


Este trabajo fue aprobado por el comité de docencia e investigación y por el comité de bioética del I.H.S.S. La recolección de la información se realizó mediante la aplicación de un instrumento elaborado, que incluyó las variables a estudiar. El instrumento que fué llenado al momento de la valoración inicial, por médico residente de posgrado de pediatría de 1, 2 y 3 año que estuviese asignado al I.H.S.S.

El seguimiento del paciente lo realizó el neurólogo pediátra en consulta externa, y estudios realizados de forma ambulatoria, fueron adicionados a los aplicado.

Una vez recolectada la información, el proceso y diseño de la base de datos se realizó en el programa Epi info versión 3.5.4.

Para el análisis estadístico se aplicaron medidas de tendencia central. Para presentar los datos se utilizó el software Microsoft Excel 2013.

La revisión metodológica estuvo a cargo del departamento de Salud Pública de la Escuela de Ciencias de la Salud del Valle de Sula, Universidad Nacional Autónoma de Honduras.

\section{RESULTADOS}

La distribución por sexo fue: 21 niñas (52.5\%) y 19 niños (47.5\%). 29 pacientes (72.5\%) de los que convulsionaron por primera vez eran menores de 2 años. Se encontró que previo al evento convulsivo, se refirieron síntomas sugestivos de aura en 5 (12.5\%) de los niños estudiados; de estos, se presentó la cefalea en $16(40 \%)$.

La duración de la primera convulsión fué menos de un minuto en 5 niños, entre uno y 5 minutos en 19 niños. 16 niños debutaron con estatus convulsivo, de los cuales 10 (62.5\%) eran menores de 2 años.

(Ver Tabla No. 1.)
Tabla No. 1: Duración de la convulsión en relación a la edad.

\begin{tabular}{llllll}
\hline $\begin{array}{l}\text { Duración } \\
\text { Iconvulsión }\end{array}$ & $\begin{array}{c}1-11 \\
\text { meses }\end{array}$ & $\begin{array}{c}12-23 \\
\text { meses }\end{array}$ & $\begin{array}{c}2-5 \\
\text { años }\end{array}$ & $\begin{array}{r}6-10 \\
\text { años }\end{array}$ & TOTAL \\
\hline < 1 minuto & 2 & 1 & 2 & 0 & 5 \\
& $40.0 \%$ & $20.0 \%$ & $40.0 \%$ & $0.0 \%$ & $12.5 \%$ \\
1a5 & $\mathbf{5}$ & 11 & 1 & 2 & 19 \\
minutos & $26.3 \%$ & $57.9 \%$ & $5.3 \%$ & $10.5 \%$ & $47.5 \%$ \\
>de5 & 9 & 1 & 5 & 1 & 16 \\
minutos & $56.3 \%$ & $6.3 \%$ & $31.3 \%$ & $6.3 \%$ & \\
& 16 & 13 & 8 & 3 & 40 \\
TOTAL & $40.0 \%$ & $32.5 \%$ & $20.0 \%$ & $7.5 \%$ & $100 \%$ \\
\hline
\end{tabular}

Fuente: Investigación; primera convulsión.

En $(n=24) 60 \%$ de los casos hubo más de un episodio convulsivo; de estos niños que presentaron una segunda convulsión, $96 \%$ se presentó dentro de las primeras 24 horas de haber convulsionado por primera vez.

Se encontró que 4 (10\%) tenían antecedente de patología perinatal, principalmente encefalopatía hipoxia isquémica e infección neonatal. Además, se encontró que 18 (45\%) tenían un familiar con antecedente de convulsiones (unos por fiebre, otros epilepsia, otros de origen desconocido). A 17 (42.5\%) niños se les realizó glucometría, encontrándose normal en 15 (88.2\%), hiperglicemia en 1 niño e hipoglicemia en el otro caso. Se le realizó electrolitos séricos a 25 (62.5\%) niños, encontrándose la hiponatremia como única alteración electrolítica en 5 (20\%) de los casos.

Se realizaron diferentes estudios de gabinete; 19 electroencefalogramas, 4 de ellos con hallazgos anormales (actividad irritativa, epilepsia), se realizaron 20 tomografías cerebrales, 5 de ellas con hallazgos anormales (lesiones, dilatación ventricular, otros). Únicamente se realizaron dos IRM cerebral, siendo una anormal; se realizaron cuatro radiografías, en una de las cuales se reportó derrame pleural como apoyo al diagnóstico de dengue en uno de los niños que convulsionó.

(Ver Tabla No. 2.) 
Tabla No. 2: Estudios de imagen realizados.

\begin{tabular}{llll}
\hline Estudios de imagen & EEG & TAC & IRM \\
\hline \multirow{2}{*}{ Anormal } & $\mathbf{4}$ & $\mathbf{5}$ & $\mathbf{1}$ \\
& $(21 \%)$ & $(40 \%)$ & $(50 \%)$ \\
Normal & $\mathbf{1 5}$ & $\mathbf{1 5}$ & $\mathbf{1}$ \\
\multirow{2}{*}{ Total } & $(79 \%)$ & $(60 \%)$ & $(50 \%)$ \\
& $\mathbf{1 9}$ & $\mathbf{2 0}$ & $\mathbf{2}$ \\
& $(100 \%)$ & $(100 \%)$ & $(100 \%)$ \\
\hline
\end{tabular}

Fuente: Investigación; primera convulsión.

Se realizó punción lumbar para estudio de Líquido Cefalorraquídeo en 8 (20\%) de los niños encontrando uno compatible con meningitis bacteriana.

Se hospitalizaron $(n=34)$ 85\% de los niños, y $(n=6)$ 15\% fueron manejados de forma ambulatoria. El tiempo de hospitalización fue de 1 a 2 días en 18 (45\%) niños, menos de 24 horas 11 (27.5\%) y más de 2 días en 11 (27.5\%) de ellos. En $(n=26) 65 \%$ de los niños se inició terapia anti comicial oral de manera permanente, siendo el ácido valproico el anticonvulsivante utilizado en 15 (57.6\%) de los niños, seguido por la fenitoína en 9 (34.6\%) de los casos. El diagnóstico más frecuente fue el síndrome convulsivo febril en 18 (45\%) de los niños, de los cuales 11 fueron síndrome convulsivo febril complejo y 7 con síndrome convulsivo febril simple.

(Ver Gráfico No 1.)

\section{Gráfico No. 1: Diagnósticos en los niños con primera convulsión.}

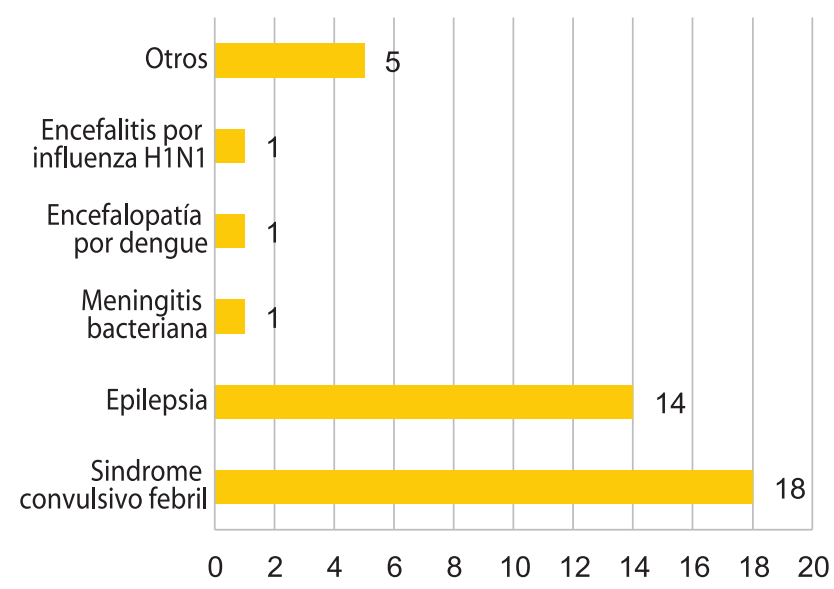

Fuente: Investigación; primera convulsión.

\section{DISCUSIÓN}

Una convulsión es un síntoma; cuando ocurre por primera vez se deberán emplear todos los recursos clínicos y paraclínicos para encontrar una patología específica etiológica. Este proceso de investigación es a veces prolongado, difícil y en ocasiones imposible. La base para llegar a un diagnóstico etiológico correcto, es la clínica; sin ésta, es imposible definir el diagnóstico.

En una crisis convulsiva existen múltiples componentes clínicos que deberán ser investigados, entre los cuales podemos mencionar: sexo, edad, presencia de fiebre, forma de inicio, vigilia o sueño, duración de crisis y del estado postictal.

En la literatura se menciona que el 8 al $10 \%$ de la población tiene el riesgo de presentar un episodio convulsivo, constituyendo el motivo de consulta en la sala de urgencias en $1-2 \%$ de las atenciones. ${ }^{(7)}$ En los pacientes con primera convulsión atendidos en el I.H.S.S. no se encontró predominio de sexo para presentar convulsiones. La edad de aparición de primera convulsión fue en los primeros dos años de vida. Este último dato discrepa de lo reportado por Hans Stroink y col, donde el rango de edad en el que es frecuente la primera convulsión es entre 6-7 años. $^{(8)}$

En un estudio realizado en el hospital Escuela de Tegucigalpa, ${ }^{(7)}$ se encontró antecedentes de convulsión en la familia en el $15 \%$ de los pacientes, a diferencia de nuestros resultados en los cuales el $45 \%$ de los niños tenían un familiar con historia de convulsión.

Existen cambios en cuanto a la clasificación de la convulsión de acuerdo a su duración y estado postictal. Se ha propuesto que una duración de 5 minutos de la crisis convulsiva es suficiente para definir el estado epiléptico. Se recomienda que las crisis convulsivas que se prolongan por más de 5 minutos se traten lo más tempranamente posible. ${ }^{(9,10)}$ En este estudio se encontró que el $40 \%$ de las convulsiones 
duraron más de 5 minutos, por lo cual se catalogaron dentro de la definición de estatus convulsivo. El $62.6 \%$ de los niños que debutaron con estatus convulsivo tenían menos de 2 años. Esta proporción es mayor a lo reportado en el Hospital Escuela de Tegucigalpa donde el $49.2 \%$ de los afectados eran menores de 3 años. ${ }^{(11)}$

Sogawa y Maytal en el 2006 reportaton que el $20 \%$ de los casos de primera convulsión recurren en las primeras 24 horas, $^{(12)}$ en el presente estudio, el $96 \%$ de los niños que presentaron recurrencia del episodio convulsivo lo presentaron dentro de las 24 horas siguientes al primer evento. La mayor interrogante de una primera crisis es, si habrá una segunda crisis o más. Este será un reto al que el médico se enfrenta y el resultado dependerá de la forma como haya sida evaluada una primer crisis.

Al ocurrir una primera crisis debemos poner en marcha todo un esquema de diagnóstico, donde deberán usarse todos los recursos clínicos y paraclínicos de la mejor calidad, y se debe tener en consideración un apoyo logístico y humano que lo garantice. En cuanto a los estudios paraclínicos como la punción lumbar $(\mathrm{PL})$, no existe evidencia científica que respalde la práctica rutinaria de PL en pacientes pediátricos con una primera crisis convulsiva. ${ }^{(13)}$ Las únicas excepciones son la aparición de crisis convulsivas en lactantes cuando hay signos clínicos que sugieran la presencia de una infección en el sistema nervioso central o en los menores de seis meses. ${ }^{(10,14)}$ En este estudio, únicamente el 20\% (8 niños) ameritó punción lumbar, encontrando citoquímica de LCR compatible con meningitis bacteriana en un caso. El valor del EEG es señalar lesiones focales, predecir la recurrencia e indicar un síndrome de epilepsia específico. No hay evidencia que apoye que el EEG deba ser realizado antes del alta del departamento de Emergencia. El estudio puede ser coordinado ambulatoriamente. ${ }^{(15)}$ En este estudio, debido a dificultades institucionales, los electroencefalogramas fueron realizados de manera ambulatoria, encontrándose anormalidades en el $21 \%$ de los estudios. El $20 \%$ de las tomografías cerebrales fueron anormales, ninguna tomografía influyó en el manejo posterior, lo cual únicamente llevó a incrementar costos, por lo que su uso debe limitarse a criterios ya establecidos para solicitar estudios de neuroimagen. ${ }^{(13,16)}$

En estudios realizados en adultos y niños, se encontró que el $30 \%$ de las primeras convulsiones fueron consecuencia de injuria cerebral y alteraciones metabólicas tóxicas. ${ }^{(17)}$ En contraste con este estudio, que se encontró como diagnóstico más frecuente el síndrome convulsivo febril en (18) $45 \%$ de los niños seguido de la epilepsia en $35 \%$ de los casos. Dentro de las causas infecciosas, se obtuvo un caso de meningitis bacteriana, un caso de encefalopatía por dengue, y un caso de encefalitis por influenza H1N1, la cual, aunque de forma poco común, se describe en la literatura como causa de convulsiones. ${ }^{(18)}$

Es recomendable que desde el primer evento convulsivo el niño sea evaluado a la brevedad posible en el servicio de urgencias de un hospital de segundo nivel. ${ }^{(19)}$ Permanecerán hospitalizados los niños que convulsionan por primera vez cuando sean menores de un año de edad, que tengan afección del estado general, datos de hipertensión endocraneana, meningismo, duración de la convulsión mayor de 15 minutos, recurrencia de convulsión en siguientes 12 horas, presenten crisis convulsivas parciales o Glasgow menor de 15.

En este estudio se observó el ingreso hospitalario en $85 \%$ de los niños con primera convulsión, con un tiempo corto de hospitalización (<48 horas en el $70.6 \%$ de los pacientes), lo que ayuda a disminuir los costos de hospitalización en un hospital de tercer nivel.

El tratamiento con medicamentos después de una primera crisis es controversial. En $65 \%$ de los niños con primera convulsión se inició tera- 
pia anti comicial oral de manera permanente, siendo el ácido valpróico el anticonvulsivante más utilizado seguido por la fenitoína. La decisión de iniciar el tratamiento debe sopesar el riesgo de otro ataque contra los riesgos de efectos secundarios del tratamiento crónico de fármacos. ${ }^{(20)}$ Se deben definir criterios para establecer en cual niño deberá iniciarse terapia anticomicial de mantenimiento, como los siguientes: menores de un año, crisis convulsivas parciales, déficit neurológico, estado epiléptico, convulsión por más de 15 minutos, estado postictal por más de 30 minutos, más de 2 convulsiones en 24 horas.

En conclusión; la población infantil con mayor riesgo de presentar primera convulsión son los menores de dos años, presentándose como estatus convulsivo en un alto porcentaje. En nuestro medio el estudio de una primer crisis convulsiva puede ser muy difícil por las limitantes del apoyo paraclínico en cantidad y calidad, teniendo en cuento que a veces la limitación también es clínica.

Se recomienda poner en marcha una estrategia que permita evaluar todas las probables causas, para poder encontrar con propiedad la etiología de una primera crisis. En una población escogida, para un estudio de primera crisis, deberá hacerse un seguimiento por lo menos de un período mínimo de dos años.

\section{BIBLIOGRAFÍA}

1. Marchezan J, Ohlweiler L, Bragatti MI, Ranzan J, Michelin M, Riesgo R. Actitud en la primera crisis convulsiva. Rev Med Hondur. 2014;82(supl. no. 2):1-108

2. Rolo YI. Convulsiones febriles en pediatría. Medwave 2009; [Revista en la internet] [citado el 21 de mayo 2015] 9(8):e4079 doi: 10.5867/ medwave.2009.08.4079. Disponible en: http://www.medwave.cl/link.cgi/ Medwave/Revisiones/RevisionClinica/4079

3. Asociación Andaluza de epilepsia. [Înternet]. Andalucía España. EPILEPSIA: MITO O REALIDAD Breve historia de la epilepsia. 2005. [Actualizado 25 mayo 2015; consultado 12 junio 2015]. Disponible en: http:// www.apiceepilepsia.org/Breve-historia-de -la-epilepsia

4. Urrestarazu E, Murie M, Viteri C. Manejo de la primera crisis epiléptica y del status en urgencias. Anales Sis San Navarra [revista en la Internet]. [citado 2015 Jun 22]. Disponible en: http://scielo.isciii.es/scielo.php? script $=$ sci_arttext $\&$ pid $=$ S1 137-6627200 8000200006\&lng=es.
5. Novoa SF. Primoconvulsión no provocada en el niño. Rev. chil. pediatr. [revista en la Internet]. 2001 Ene [citado 2015 Jun 23] ; 72 (1): 6-11. doi.org/10.4067/S0370-41062 001000100002. Disponible en: http://www. scielo.cl/scielo.php?script=sci_arttext\& pid=S0370-41062001000100002\& Ing=es

6. Gómez AJ, Bellas LP. Nueva clasificación de las epilepsias de la Liga Internacional contra la Epilepsia (ILAE): ¿un paso en dirección equivocada? Rev Neurol 2011; [Citado 13 de mayo de 2015] 52 (9):541-547. Disponible en: http://www.neurologia.com/pdf/ Web/5209/bf090541.pdf

7. Lara Castillo M, Molinero M.Caracterización del síndrome convulsivo en menores de 12 años del 2004 al 2006. Honduras Pediátrica. 2007; XXVII(3)

8. Stroinka H, Brouwerb OF, Frans Artsd W, Geertsc AT, Boudewyn PAC, Donselaara CA. The first unprovoked, untreated seizure in childhood: a hospital based study of the accuracy of the diagnosis, rate of recurren ce, and long term outcome after recurrence. 
Dutch study of epilepsy in childhood. J Neurol Neurosurg Psychiatry 1998 [Citado 13 mayo de 2015]; 64:595-600 doi:10.1136/ jnnp.64.5.595. Disponible en: http://jnnp. bmj.com/content/64/5/595

9. Rubio DF, Reséndiz AJ, Sentiés MH, Alonso VM, Ramos PJ. Programa Prioritario de Epilepsia. México: Sector Salud; 2007.

10. Corral Ansaa I, Herrero Meseguera J, Falip centellas M, Aiguabella-Macau M. Estatus epiléptico. Med intensiva. 2008; 32(4): 174-82

11. Valladares Cerrato V, Samra J, Molinero M. Caracterizar al paciente con estatus convulsivo en el área Pediátrica, atendidos en la emergencia del bloque materno infantil del hospital escuela. Honduras Pediátrica 2003; XXIII(2).

12. Sogawa Y, Maytal J. 2006 Emergency department admission of children with unprovoked seizure: recurrence within 24 hours. Pediatr Neurol. 2006 [Citado, 22 noviembre 2014]; 35(2):98-101. Disponible en: http://www.ncbi.nlm.nih.gov/pubmed/ 16876004

13. Baraibar Ana, Castro Mercedes, Cerisola Alfredo, Martínez Adriana, Misa Adriana, Montano Alicia, et al. Guía de práctica clínica para el diagnóstico y tratamiento de la primera convulsión en apirexia, no provocada, en niños entre un mes y 14 años: (versión abreviada). Arch. Pediatr. Urug. [revista en la Internet]. 2004 [citado 2015 Jun 23] ; 75(3): 263-272. Disponible en: http:// www.scielo.edu.uy/scielo.php?script $=$ sci arttext\&pid=S1688-12492004000300008 \&lng=es.

14. Batra P, Gupta S, Gomber S, Saha A. Predictors of meningitis in children presenting with first febrile seizures. Pediatr. Neurol. 2011; 44 (1):35-9. doi: 10.1016/j.pediatrneu rol.2010.07.005.

15. Sociedad Andaluza de epilepsia. Guía andaluza de epilepsia 2009. Diagnóstico y tratamiento de la epilepsia en niños y adultos. Madrid: Emisa; 2009.

16. Kalnin AJ, Fastenau PS, de Grauw TJ, Musick BS, Perkins SM, Johnson CS, et al. MR Imaging Findings in Children with First Recognized Seizure. Pediatr. Neurol. 2008; 39(6): 404-414. doi: 10.1016/j.pediatrneu rol. 2008.08.008

17. Polhmann B, Beghi E, Camfield C, Camfield P. The first seizure and management in adult and children. BMJ. [Revista en internet] 2006 [Citado 13 diciembre 2014]; 332(7537): 339-342. doi: 10.1136/bmj.332. 7537.339. Disponible en: http://www.nc bi.nlm.nih.gov/pmc/articles/PMC1363913/

18. Kwong KL, Lam SY, Que TL, Wong SN. Influenza $A$ and febrile seizures in child hood. Pediatric. Neurology. 2006; 35 (6): 395-399. DOI: 10.1016/j.pediatrneurol.2006. 07.005 .

19. Scottish Intercollegiate Guidelines Network. Diagnosis and management of epilepsies in childrens and young people. A national clinical guidelines. Escosia; 2005.

20. Hirtz D, Berg A, Bettis D, Camfield C, Camfield $\mathrm{P}$, Crumrine $\mathrm{P}$, et al. Practice parameter: treatment of the child with a first unprovoked seizure: Report of the Quality Standards Subcommittee of the American Academy of Neurology and the Practice Committee of the Child Neurology Society. Neurology. 2003 [citado 11 noviembre 2014]; 60(2):166-75. Disponible en: http:// www.ncbi.nlm.nih.gov/pubmed/12552027 